Check for updates

Cite this: RSC Adv., 2019, 9, 16431

Received 3rd April 2019

Accepted 20th May 2019

DOI: $10.1039 / c 9 r a 02494 h$

rsc.li/rsc-advances

\section{High-aspect-ratio mushroom-like silica nanopillars immersed in air: epsilon-near-zero metamaterials mediated by a phonon-polaritonic anisotropy $\dagger$}

\author{
Kota Ito, ${ }^{a}$ Yuri Yamada, (iD *a Atsushi Miura ${ }^{a}$ and Hideo lizuka (D) *b
}

Epsilon-near-zero metamaterials offer opportunities for intriguing electromagnetic-wave phenomena. Here we experimentally demonstrate that silica perpendicular nanopillars immersed in air exhibit a uniaxial epsilon-near-zero response mediated by phonon polaritons in the mid-infrared range. Unique mushroom-shaped heads on nanopillars play a crucial role to realize $\mathrm{SiO}_{2}$ metamaterials over a large area in our self-assembled fabrication process with block copolymers, polystyrene-blockpoly(dimethylsiloxane) (PS-b-PDMS). $\mathrm{SiO}_{2}$ nanopillars having heights of $80 \mathrm{~nm}, 200 \mathrm{~nm}$, and $300 \mathrm{~nm}$ (aspect ratios up to $\sim 13$ ) are obtained after calcination at $450{ }^{\circ} \mathrm{C}$ and the electromagnetic responses are evaluated using a mid-infrared ellipsometric apparatus. For nanopillars with $200 \mathrm{~nm}$ height, the permittivity of the perpendicular component $\varepsilon_{\perp}$ approaches to near zero (0.2) while the parallel component $\varepsilon_{\|}$shows a value of 1.8. The measured uniaxial epsilon-near-zero responses are excellently reproduced by the effective medium theory. Our results, therefore, indicate that $\mathrm{SiO}_{2}$ nanopillars/air uniaxial epsilon-near-zero metamaterials in the mid-infrared range can be amenable to large scale fabrication.

\section{Introduction}

Researchers have designed electromagnetic metamaterials with unconventional permittivity and permeability values in various frequency ranges. Epsilon-near-zero metamaterials are unique class of materials, which enable the permittivity values lower than that of the free-space. ${ }^{\mathbf{1}}$ They have realized various applications ranging from perfect imaging systems, ${ }^{2}$ waveform shaping, ${ }^{3}$ light tunnelling, ${ }^{4,5}$ perfect absorption, ${ }^{6}$ and spontaneous emission enhancement. ${ }^{7}$ Sometimes epsilon-near-zero metamaterials are designed to have anisotropic ${ }^{8,9}$ or more specifically uniaxial permittivities, ${ }^{\mathbf{1 0}}$ i.e., the permittivity component in the direction perpendicular to the film is different from that in the direction parallel to the film. Metal and dielectric films are stacked alternately to achieve an epsilon-near-zero permittivity in the parallel direction, and that has been utilized to control thermal emission ${ }^{\mathbf{1 1}}$ and optical response. ${ }^{12}$ Alternatively, metallic nanopillar arrays can achieve a uniaxial anisotropy with an epsilon-near-zero permittivity in the perpendicular direction. One pioneering work is $\mathrm{Au}$

\footnotetext{
${ }^{a}$ Toyota Central Research \& Development Laboratories, Inc., 41-1 Yokomichi, Nagakute, Aichi, 480-1192, Japan.E-mail: e4610@mosk.tytlabs.co.jp

${ }^{b}$ Toyota Research Institute of North America, Toyota Motor North America, Ann Arbor, Michigan 48105, USA. E-mail: hideo.iizuka@toyota.com

$\dagger$ Electronic supplementary information (ESI) available: SEM images of $\mathrm{SiO}_{2}$ dot arrays without $\mathrm{O}_{2}$ plasma process, XPS depth profiles, depolarization factors, and the calculated Lorentz parameters. See DOI: 10.1039/c9ra02494h
}

nanorod arrays obtained by electroplating in porous anodic alumina oxide (AAO) templates..$^{13-16}$ The $\mathrm{Au}$ nanorods also realize the polarization converters ${ }^{\mathbf{1 7}}$ because the phase delay between p-polarized (perpendicular) and s-polarized (parallel) light is significantly different.

Surface phonon polaritons observed in polar materials, such as hexagonal boron nitride (hBN), $\mathrm{SiC}$, and $\mathrm{SiO}_{2}$, provide strong light-matter interaction in the mid-infrared wavelength range. ${ }^{18-24}$ Of these materials, $\mathrm{SiO}_{2}$ is a prominent candidate due to ease of synthesis and its numerous potential applications. $\mathrm{SiO}_{2}$ has been widely explored for various application scenarios, such as thermal emission control, ${ }^{25,26}$ superlenses, ${ }^{27,28}$ and biosensing. ${ }^{29}$ Particularly, for thermal emission, when one of the components in such metamaterials is air with small thermal conductivity or vacuum with no thermal conductivity, one can expand design variation in tailoring electromagnetic waves.

In spite of its importance for numerous applications, the mid-infrared device fabrication often suffers from complicated top-down procedures, which are relatively expensive and timeconsuming. Self-assembly of block copolymers provides periodically ordered patterns on substrates with relatively simple and low-cost manners. ${ }^{30-32}$ In these block copolymers, dissimilar polymer chains are covalently linked to each other, and form microphase-separated structures at a molecular level. The long-range periodicity of the obtained microphase-separated structures is greatly enhanced by various external fields, such as vapor ${ }^{33,34}$ or thermal ${ }^{35}$ annealing. The formed microphase has been utilized as nanomasks for the subsequent 
microfabrication, ${ }^{36}$ profiting from the fact that the pattern feature size is well below the conventional photolithography. A number of well-ordered microphase structures have been realized including in-plane cylinders, ${ }^{37,38}$ perforated lamella, and vertical cylinders. ${ }^{39,40}$ Vertical cylinders based on the selfassembly of block copolymers are attracting of great interest because they can be a viable solution to fabricate metamaterials, leading to observe epsilon-near-zero behaviours.

Previously, we have reported a fabrication of $\mathrm{SiO}_{2}$ perpendicular pillar arrays from self-assembled block copolymers, polystyrene-block-poly(dimethylsiloxane) (PS- $b$-PDMS), using solvent annealing and several etching steps. ${ }^{41}$ We have speculated that the unique mushroom-shaped heads on nanopillars play a crucial role to maintain a hexagonal regularity during removal of organic components, which was yielded by $\mathrm{O}_{2}$-RIE. The obtained perpendicular pillars are expected to be a platform for $\mathrm{SiO}_{2}$ metamaterials or nanophotonic devices, but its possibility has not been investigated. In this paper, we report a uniaxial epsilon-near-zero behaviour enabled by selfassembled silica nanopillar arrays immersed in air. The electromagnetic response is evaluated at each etching step and the route for $\mathrm{SiO}_{2}$ metamaterials is clearly presented. Mid-infrared ellipsometry has been performed to characterize the effective permittivity of the $\mathrm{SiO}_{2}$ nanopillar arrays. The results validate that self-assembly of block copolymers enables to fabricate metamaterials, having a uniaxial epsilon-near-zero characteristic. The measured uniaxial epsilon-near-zero responses are excellently reproduced by the effective medium theory using retrieved permittivity parameters. We have to mention that there are several pioneering works on manipulating heat flow. Recently, thermal emission and conduction, ${ }^{42}$ or thermal conduction and convection ${ }^{\mathbf{4 3}}$ are simultaneously engineered. They have demonstrated sophisticated ways to control two thermal properties out of three (i.e. thermal emission, conduction and convection) and realized promising thermal designs. In this work, we focus on engineering electromagnetic responses (thermal emission) using $\mathrm{SiO}_{2}$ nanopillar arrays with unique mushroom-shaped heads. It would be a great challenge to control two thermal properties with the specific $\mathrm{SiO}_{2}$ metamaterials, however the idea is beyond the scope of this study. The effort to utilize our $\mathrm{SiO}_{2}$ metamaterials is underway to propose an advantageous thermal and optical designs.

\section{Experimental}

\subsection{Pattern formation of mushroom-like silica nanopillars}

The overall preparation process of $\mathrm{SiO}_{2}$ cylinder arrays is shown in Fig. 1. Firstly, cylindrical patterns have been formed from the spin-casted film with phase composition of 3-8 wt $\%$ PS- $b$-PDMS dissolved in THF. After solvent annealing in chloroform vapor for $90 \mathrm{~min}, \mathrm{CF}_{4} / \mathrm{O}_{2} \mathrm{RIE}$ (reactive ion etching) has been applied under a $\mathrm{CF}_{4}$ flow rate of $0.1 \mathrm{~Pa} \mathrm{~m}^{3} \mathrm{~s}^{-1}$ and an $\mathrm{O}_{2}$ flow rate of 0.17 $\mathrm{Pa} \mathrm{m}^{3} \mathrm{~s}^{-1}$ for $60 \mathrm{~s}$ at a power of $200 \mathrm{~W}$ and chamber pressure of $16 \mathrm{~Pa}$ in the case of route $\mathrm{B}$ in Fig. 1 . With regard to $\mathrm{CF}_{4} / \mathrm{O}_{2} \mathrm{RIE}$, the method established by Son et al. ${ }^{39}$ was modified. $\mathrm{O}_{2}$ plasma treatment has been applied to the film using a plasma reactor (PR300, Yamato Science Co. Ltd.) at $100 \mathrm{~W}$ under an $\mathrm{O}_{2}$ flow rate of $0.08 \mathrm{~Pa} \mathrm{~m}^{3} \mathrm{~s}^{-1}$, followed by calcination at $450{ }^{\circ} \mathrm{C}$. The same $\mathrm{O}_{2}$ plasma process has been carried out without $\mathrm{O}_{2}$ RIE on the film fabricated via route $\mathrm{A}$. For route $\mathrm{C}$, the film was calcined at $450{ }^{\circ} \mathrm{C}$ after continuous $\mathrm{CF}_{4} / \mathrm{O}_{2} \mathrm{RIE}$ (without $\mathrm{O}_{2}$ plasma). RIE system (RIE-10NR, Samco Inc.) was employed in this study.

\subsection{Ellipsometric measurement}

We employed ellipsometric technique to evaluate $\mathrm{SiO}_{2}$ nanopillar arrays with the unique mushroom-shaped heads. Ellipsometry is a well-established method to characterize optical responses of thin films. ${ }^{44}$ In ellipsometry, a difference in light reflection of $\mathrm{p}$ - and s-polarizations is detected as the changes in a polarization state. The samples were measured by a midinfrared ellipsometric spectrometer (IR-VASE, J. A. Woollam). The measured results were represented by two values, namely $\Psi$ and $\Delta$ as:

$$
\begin{gathered}
\Psi=\tan ^{-1}\left|\frac{r_{\mathrm{p}}}{r_{\mathrm{s}}}\right|, \\
\Delta=\arg \left(\frac{r_{\mathrm{p}}}{r_{\mathrm{s}}}\right),
\end{gathered}
$$

where $r_{\mathrm{p}}$ and $r_{\mathrm{s}}$ are the Fresnel reflection coefficients in $\mathrm{p}$ - and spolarizations. $\Psi$ and $\Delta$ represent relative amplitude and phase difference of the p-polarized reflection coefficient to the spolarized counterpart. Thus the tilt of the samples and other artifacts are easily compensated in measurement results and how the permittivities of our samples relate to the two polarizations is observed through eqn (1) and (2). The absolute values of $\Psi$ and $\Delta$ do not have significant meaning, thus an optical model is required to analyse measurement data. In this paper, we mainly discuss the frequency responses of our samples with eqn (1) and (2) after showing the p-polarized reflection coefficients (Fig. 2).

\subsection{Characterization}

SEM images of surface and cross-sectional views were obtained using field-emission scanning electron microscopes (Hitachi S4800 and S-5500, respectively). FT-IR (Fourier-transformed infrared) spectroscopy was conducted on an FT-IR spectrometer (Nicolet iS50, Thermo Fisher Scientific, Inc.) to investigate compositional change of the nanopillars during the fabrication steps. The incident angle from the surface normal was changed from $30^{\circ}$ to $60^{\circ}$ at every $10^{\circ}$. The finite integration-technique based simulator CST Microwave Studio was utilized in the frequency domain for electromagnetic simulations of our nanostructures. The electromagnetic fields were excited and monitored by Floquet ports, and the reflection coefficients were calculated. From eqn (1) and (2) with the simulated reflection coefficients, we obtained $\Psi$ and $\Delta$.

\section{Results and discussion}

\subsection{Changes of electromagnetic status depending on process}

Firstly, we measured FTIR spectra on $\mathrm{SiO}_{2}$ nanopillars obtained thorough route $\mathrm{B}$ in Fig. 1 to investigate the electromagnetic 


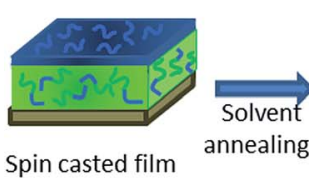

Si substrate

PS

Quartz like $\mathrm{SiO}_{2}$

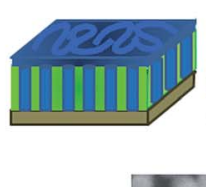

PDMS

$\mathrm{SiO}_{2}$

.
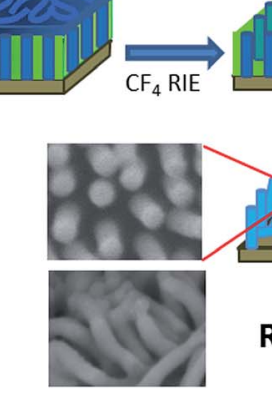

Route A

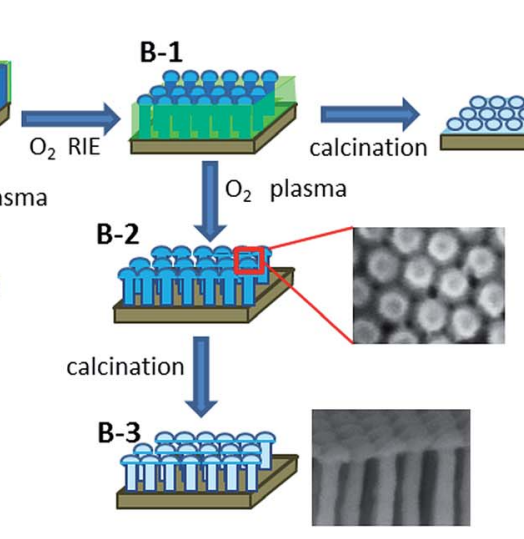

Route B

Fig. 1 Schematic of fabrication routes of $\mathrm{SiO}_{2}$ nanopillar arrays depending on the sequential etching process.

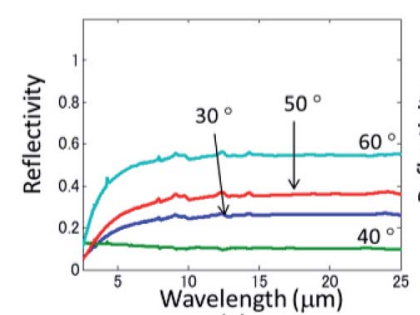

(a)

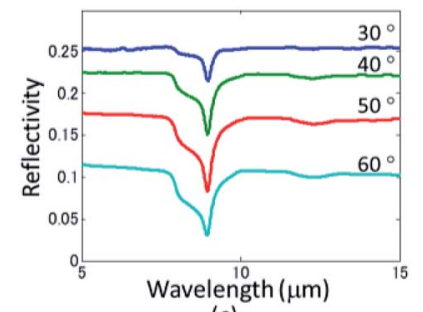

(c)

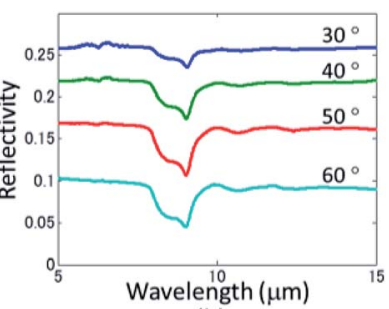

(b)
Fig. 2 P-polarized reflection spectra using FTIR spectroscopy during transition after (a) $\mathrm{CF}_{4}$ and $\mathrm{O}_{2} \mathrm{RIE}$, followed by (b) $\mathrm{O}_{2}$ plasma, and (c) calcination at $450{ }^{\circ} \mathrm{C}$.

status at each etching step. Fig. 2 shows the obtained results of $\mathrm{SiO}_{2}$ nanopillars fabricated from a $5 \mathrm{wt} \%$ PS- $b$-PDMS solution via route B in Fig. 1.

In Fig. 2(a), flat reflection spectra were observed in the wavelength range from 7 to $20 \mu \mathrm{m}$ after $\mathrm{CF}_{4}$ and $\mathrm{O}_{2}$ RIE (B-1 in Fig. 1). Then in Fig. 2(b), a broad dip has emerged at around 8.5 $\mu \mathrm{m}$ after $\mathrm{O}_{2}$ plasma (B-2 in Fig. 1) at all angles. The obtained dips correspond to fused quartz phonons, which induce an epsilon-near zero response. But at this stage, a hallo-like dent was also detected at around $11 \mu \mathrm{m}$, indicating amorphous silica structure remains to some extent. Upon calcination at $450{ }^{\circ} \mathrm{C}$ (Fig. 2(c)), the dips at around $8.5 \mu \mathrm{m}$ become sharper and the hallo-like dent patterns at $11 \mu \mathrm{m}$ disappear because the permittivity of the $\mathrm{SiO}_{2}$ nanopillars approaches to that of fused quartz. These measured results indicate that calcination step, which is newly added in this study, enables fabrication of the fused quartz-like $\mathrm{SiO}_{2}$ nanopillars from the self-assembly of PS$b$-PDMS. Then, we investigate morphological change of $\mathrm{SiO}_{2}$ nanopillars upon the sequential etching steps using SEM (Fig. 3). As was indicated in the previous report, $\mathrm{O}_{2}$ RIE partially melts the thin surface layer of PDMS pillars and yields mushroom-like heads, which is supposed to play a key role to maintain a hexagonal regularity during removal of organic components by $\mathrm{O}_{2}$ plasma. ${ }^{41}$ The morphology is well observed in cross-sectional SEM images (Fig. 3(a, c and e)) and more detailed explanation for the formation of mushroom-like heads can be found in elsewhere. ${ }^{45}$ We newly find that mushroom-like heads are effective to maintain the regularity of the pillar structure even at $450{ }^{\circ} \mathrm{C}$ calcination. No significant degradation in the hexagonal regularity during calcination is seen in Fig. 3(e) and an average height of $200 \mathrm{~nm}$, corresponding to an aspect ratio of 8.7 unveils for the fused quartz-like $\mathrm{SiO}_{2}$ nanopillars.
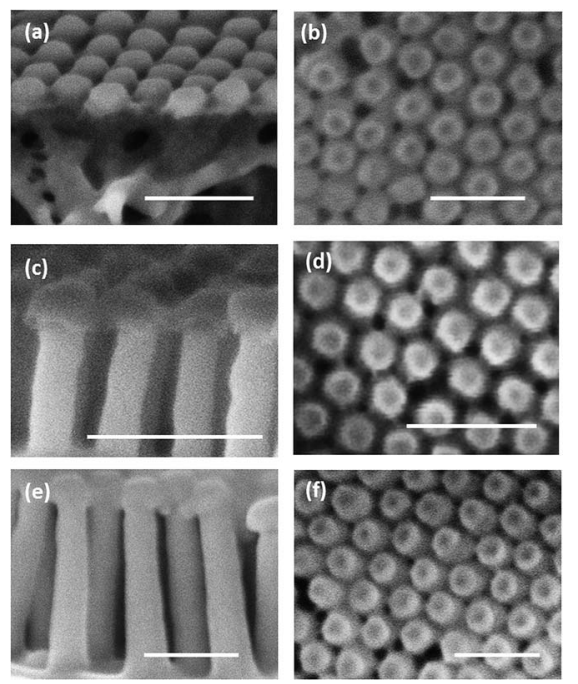

Fig. 3 (a, $c$ and e) Cross-sectional and ( $b$, $d$ and $f$ ) top view SEM images showing the emergence and maintenance of perpendicular cylinders from the $5 w t \%$ PS- $b$-PDMS solution via route B in Fig. 1. Images were taken ( $a$ and b) after $\mathrm{CF}_{4}$ and $\mathrm{O}_{2}$ RIE (B-1 in Fig. 1), (c and d) after $\mathrm{O}_{2}$ plasma for $240 \mathrm{~s}$ (B-2 in Fig. 1), and (e and f) after calcination at $450{ }^{\circ} \mathrm{C}$ (B-3 in Fig. 1). The scale bars indicate $100 \mathrm{~nm}$, respectively. 
Here, we investigate other conceivable routes for $\mathrm{SiO}_{2}$ metamaterials consisting from nanopillar arrays (route A and route $\mathrm{C}$ in Fig. 1). In the route $\mathrm{A}$, the pillar arrays are fabricated without $\mathrm{O}_{2}$ RIE, leading to significant deterioration of the hexagonal regularity upon the $\mathrm{O}_{2}$ plasma treatment, which is shown in Fig. 4. In the case that the $\mathrm{O}_{2}$ plasma process has been conducted with a short duration (60 s), PS remains in the bottom of the pillars as shown in Fig. 4(a), which acts as a foundation to maintain the hexagonal regularity, instead of adhesion between the adjutant mushroom-like heads (Fig. 3). Then we have lengthened the duration of $\mathrm{O}_{2}$ plasma $(120 \mathrm{~s})$, and found that the regularity of the remaining pillar arrays was significantly lowered as the organic remnants were removed (Fig. 4(b)). It should be noted that the higher the PDMS pillars, the more severely the regularity of nanopillar arrays is deteriorated. The observation in this study verified our concept, that the mushroom-like heads are essential on fabrication of the fused quartz-like $\mathrm{SiO}_{2}$ nanopillars using self-assembly of PS- $b$ PDMS. One may doubt a necessity of the $\mathrm{O}_{2}$ plasma treatment to remove organic remnants and fully oxide the PDMS to the fused quartz. Calcination at $450{ }^{\circ} \mathrm{C}$ immediately after the continuous RIEs (route $\mathrm{C}$ in Fig. 1) can provide a mushroom-like heads as well as remove the organic components. However, nanopillars with high aspect ratios are not obtained. Nanopillars seem to collapse and form dot arrays upon calcination without the $\mathrm{O}_{2}$ plasma process (Fig. $\mathrm{S} 1$ in ESI $\dagger$ ). $\mathrm{O}_{2}$ plasma is frequently used for surface modification of plastics, and increase of oxygen content on the surface was reported. ${ }^{46}$ The thickness of surface oxide layers was studied using XPS depth-profiling technique (Fig. S2 in ESI $\dagger$ ). The thick oxide layer was yielded upon the $\mathrm{O}_{2}$ plasm process, which can act as a protecting substance during calcination at $450{ }^{\circ} \mathrm{C}$. It is obvious that the route B in Fig. 1 is an only solution to fabricate $\mathrm{SiO}_{2}$ nanopillar arrays having a hexagonal regularity and fused-quartz-like electromagnetic states.

\subsection{Ellipsometry measurement and prediction of the optical anisotropy}

We evaluated the fused-quartz-like nanopillar arrays fabricated from the 5\% PS- $b$-PDMS solution. Ellipsometrical parameters $\Psi$ and $\Delta$ were measured by the mid-infrared ellipsometry apparatus and the results were depicted in Fig. 5. Data from 5
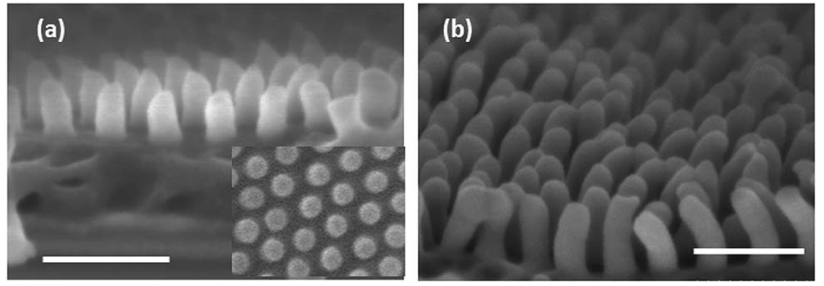

Fig. 4 Cross-sectional SEM images showing the emergence of straight perpendicular cylinders from a 3 wt $\%$ PS- $b$-PDMS solution via route A in Fig. 1. $\mathrm{O}_{2}$ plasma treatment was conducted for (a) $60 \mathrm{~s}$ and (b) $120 \mathrm{~s}$. The inset in (a) is a top-view image of the straight shape pillars. The scale bars indicate $100 \mathrm{~nm}$.

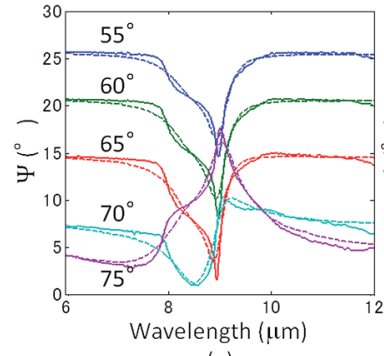

(a)

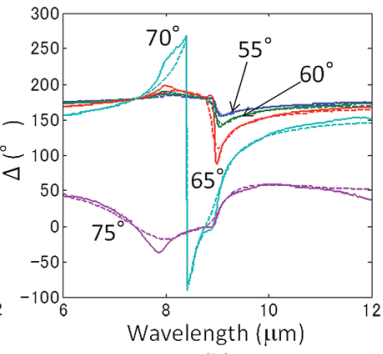

(b)
Fig. 5 Ellipsometrically measured and calculated parameters $\Psi$ (a) and $\Delta$ (b). Data are obtained at angles of $55^{\circ}$ (blue line), $60^{\circ}$ (green line), $65^{\circ}$ (red line), $70^{\circ}$ (cyan line), and $75^{\circ}$ (purple line), respectively. Solid curves are measured results and dotted curves are predicted results by the effective medium theory.

different angles, namely $55^{\circ}, 60^{\circ}, 65^{\circ}, 70^{\circ}$, and $75^{\circ}$ have been presented. In the spectra for $\Psi$, the peaks are negative in the case that the incident angle is smaller than the Brewster angle of the silicon and air interface $\left(73^{\circ}\right)$. A strong resonant mode was clearly identified at around $9 \mu \mathrm{m}$ in Fig. 5(a) which was induced by phonon polariton excitations. The changes in $\Psi$ and $\Delta$ come from the phonon polariton excitation in our nanopillars that is seen in Fig. 2. As pointed out in the previous section, the clear dips are detected in the reflectivity (Fig. 2) where the resonant mode is observed in Fig. 5. Note that the depolarization factors measured in the optical ellipsometry are also shown in Fig. S3 in ESI, $\dagger$ and it is smaller than $2 \%$ in the wavelength range of interest.

Then, we will retrieve a uniaxial permittivity from the measured $\Psi$ and $\Delta$ by utilizing the effective medium theory. The permittivity of $\mathrm{SiO}_{2}\left(\varepsilon_{\mathrm{Q}}\right)$ is expressed by the Lorentz model.

$$
\varepsilon_{\mathrm{Q}}=\varepsilon_{\infty}+A_{1} \frac{\omega_{01}{ }^{2}}{\omega_{01}{ }^{2}-\omega^{2}-\mathrm{i} \Gamma_{1} \omega}+A_{2} \frac{\omega_{02}{ }^{2}}{\omega_{02}{ }^{2}-\omega^{2}-\mathrm{i} \Gamma_{2} \omega},
$$

where $\omega$ is the angular frequency, $\varepsilon_{\infty}$ is the permittivity at infinite frequency, $A_{1,2}$ is the oscillator strength, $\omega_{01,02}$ is the resonance frequency, and $\Gamma_{1,2}$ is the damping rates for twopoles. Here, we regard the arrays of $\mathrm{SiO}_{2}$ nanopillars as a thin film with an anisotropic permittivity according to the effective medium theory. The permittivity components in the directions of perpendicular $\left(\varepsilon_{\perp}\right)$, and parallel $\left(\varepsilon_{\|}\right)$to the film surface are given by $^{47}$

$$
\begin{gathered}
\varepsilon_{\perp}=f \varepsilon_{\mathrm{Q}}+\varepsilon_{\mathrm{A}}(1-f), \\
\varepsilon_{\|}=\varepsilon_{\mathrm{A}} \frac{\varepsilon_{\mathrm{Q}}(1+f)+\varepsilon_{\mathrm{A}}(1-f)}{\varepsilon_{\mathrm{Q}}(1-f)+\varepsilon_{\mathrm{A}}(1+f)},
\end{gathered}
$$

where $f$ is the volume fraction of the $\mathrm{SiO}_{2}$ nanopillars within the film, and $\varepsilon_{\mathrm{A}}$ is the permittivity of air. Together with these values and the height of the $\mathrm{SiO}_{2}$ nanopillars estimated from the SEM observation, we fit a set of permittivity parameters to explain the experimental results (Table $\mathrm{S} 1$ in ESI $\dagger$ ). The calculated $\Psi$ and $\Delta$, based on the fitted parameters, are shown as the dotted lines in Fig. 5. The solid and the dotted curves agree quite well, 
indicating that the experimental results are well reproduced by the calculated values over the entire substrate.

The predicted permittivities based on the effective medium theory are shown in Fig. 6. We observe an anisotropic nature in the permittivity in Fig. 6(a), where the perpendicular component $\varepsilon_{\perp}$ approaches 0.2 with a parallel component $\varepsilon_{\|}$of 1.8 . The results validate that self-assembly of block copolymers enables to realize a phonon-polaritonic anisotropy, which facilitates uniaxial epsilon-near-zero metamaterials. We have also predicted intrinsic permittivity $\varepsilon_{\mathrm{Q}}$ as shown in Fig. 6(b). It is clear that the resonant wavelength of the obtained nanopillars is almost identical to that of the reported fused quartz, though the resonant amplitude of the nanopillars is smaller than the reported value. This phenomenon may come from the low calcination temperature in this study $\left(450{ }^{\circ} \mathrm{C}\right)$, which leads to a weaker $\mathrm{SiO}_{2}$ bond than nominal fused quartz substrate.

\subsection{Ellipsometric measurements on nanopillars having heights of 80 and $300 \mathrm{~nm}$}

The $\mathrm{SiO}_{2}$ nanopillars with other heights have been also measured by the mid-infrared ellipsometric apparatus to verify our calculations. Changing the content of PS- $b$-PDMS in the spin-casting solution allows us to tune the height of the $\mathrm{SiO}_{2}$ nanopillars easily via route $\mathrm{B}$ in Fig. 1 . As was mentioned in the previous section (3.2), the $\mathrm{SiO}_{2}$ nanopillars with a height of around $200 \mathrm{~nm}$ are obtained from the $5 \mathrm{wt} \%$ PS- $b$-PDMS solution. Meanwhile, the compositional weight percentages of $3 \mathrm{wt} \%$ and $8 \mathrm{wt} \%$, respectively, realize the pillars whose heights are $80 \mathrm{~nm}$ and $300 \mathrm{~nm}$ as shown in Fig. 7(a) and (b). In both cases, perpendicular cylinder arrays having mushroom-like heads are clearly observed even after $450{ }^{\circ} \mathrm{C}$ calcination. An aspect ratio as high as 13 is achieved from the $8 \mathrm{wt} \%$ PS- $b$-PDMS solution after calcination (Fig. 7(b)).

The permittivities for the films fabricated from 3 and $8 \mathrm{wt} \%$ of PS- $b$-PDMS solutions are modelled by the same manner retrieved in the film prepared from the $5 \mathrm{wt} \%$ solution.

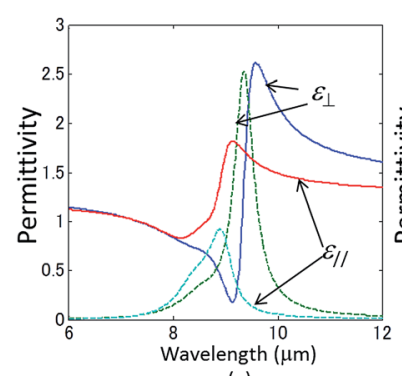

(a)

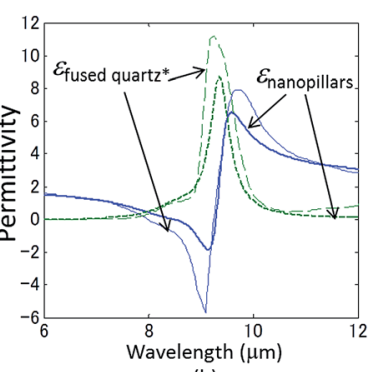

(b)
Fig. 6 Predicted permittivities of $\mathrm{SiO}_{2}$ nanopillars. (a) Perpendicular component (blue solid curve: real part, and green dashed curve: imaginary part) and the parallel component (red solid curve: real part, and cyan dashed curve: imaginary part) of the uniaxial permittivity of the $\mathrm{SiO}_{2}$ nanopillars. (b) Intrinsic permittivity of the $\mathrm{SiO}_{2}$ nanopillars (thick blue curve: real part, and thick green dashed curve: imaginary part). The permittivity of fused quartz in ref. 48 is shown for comparison (thin blue curve: real part, and thin green dashed curve: imaginary part).
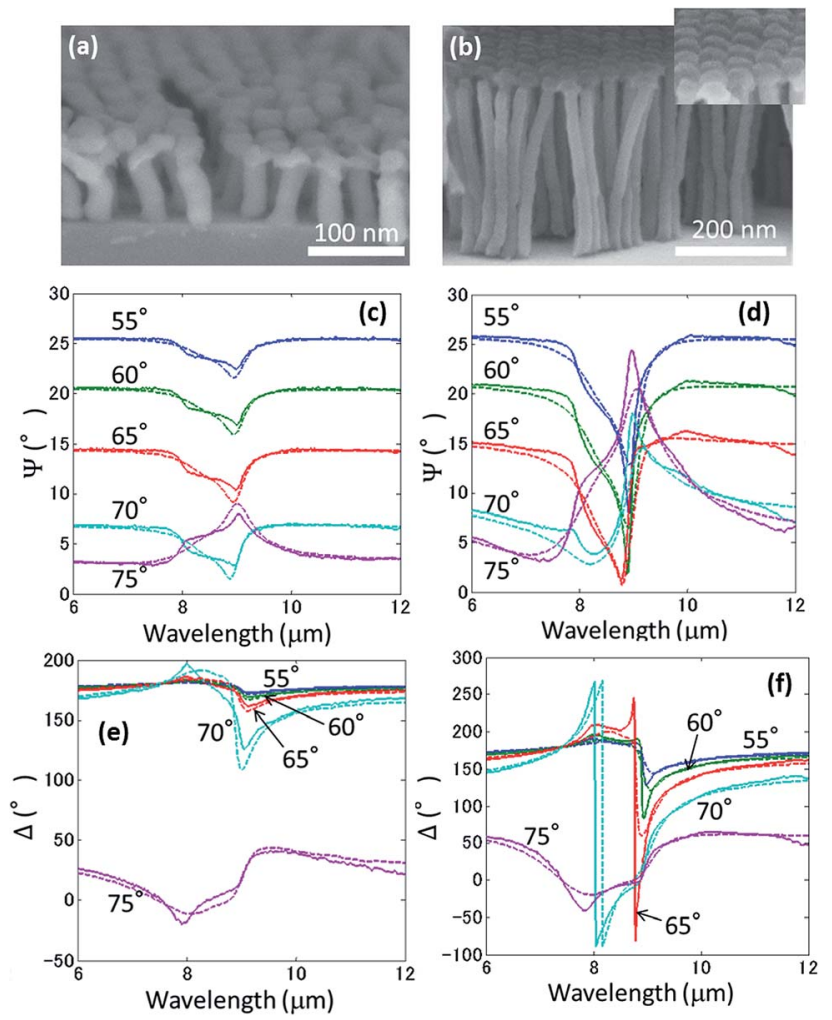

Fig. $7 \mathrm{SEM}$ images of the $\mathrm{SiO}_{2}$ nanopillars fabricated from (a) 3 wt\% and (b) 8 wt\% PS-b-PDMS solutions. Inset in (b) represents the adhesion between mushroom-like heads of adjacent pillars. Ellipsometrically measured and calculated parameters $\Psi$ (c and d) and $\Delta$ (e and f) for films fabricated from $3 \mathrm{wt} \%$ ( $c$ and e) and 8 wt\% (d and f) PS- $b-$ PDMS solutions. Data are obtained at angles of $55^{\circ}$ (blue line), $60^{\circ}$ (green line), $65^{\circ}$ (red line), $70^{\circ}$ (cyan line), and $75^{\circ}$ (purple line), respectively. Solid curves are measured results and dotted curves are predicted results by the effective medium theory.

Ellipsometrically measured parameters $\Psi$ and $\Delta$ are compared with those obtained from the predicted models (Fig. 7(c-f)). The results show that the modelled permittivity well explains the obtained cylinder arrays with each height. It is well observed that the measured peaks and the dips become steeper as the pillar height increases due to the enhancement of the anisotropy. The experimental results are well reproduced on calculations for $\mathrm{SiO}_{2}$ nanopillars with different heights, which corroborates our predicted model strongly.

Importance of the high-aspect ratio upon optical anisotropy which can expand material designs is shown in previous discussion. Additionally, we would like to compare directly the impact of the aspect ratio of the $\mathrm{SiO}_{2}$ nanopillar arrays on the electromagnetic responses. Uniaxial permittivity media provide the feature of different wavevectors for $\mathrm{p}$ - and s-polarized waves. Using the retrieved permittivity components in Fig. 6, we calculate the optical lengths of $\mathrm{p}$ - and s-polarized waves along the nanopillars. Fig. 8 shows the relative optical length of the ppolarized wave to the s-polarized wave, the real part of $\gamma_{\mathrm{p}} h-\gamma_{\mathrm{s}} h$ with pillar heights of $h=80 \mathrm{~nm}, 200 \mathrm{~nm}$, and $300 \mathrm{~nm}$, respectively. In this relation, the perpendicular wavevector 


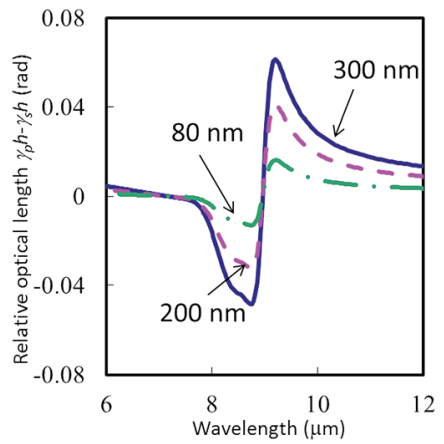

Fig. 8 Relative optical length of the $p$-polarized wave to the spolarized wave in the nanopillar array with heights of $80 \mathrm{~nm}, 200 \mathrm{~nm}$ and $300 \mathrm{~nm}$, respectively. Data are calculated at an angle of $65^{\circ}$.

components for p-polarization $\left(\gamma_{\mathrm{p}}\right)$ and s-polarization $\left(\gamma_{\mathrm{s}}\right)$ are expressed as:

$$
\begin{gathered}
\gamma_{\mathrm{p}}=\sqrt{\varepsilon_{\|} k_{0}^{2}-\left(\varepsilon_{\|} / \varepsilon_{\perp}\right) \beta^{2}}, \\
\gamma_{\mathrm{s}}=\sqrt{\varepsilon_{\|} k_{0}^{2}-\beta^{2}},
\end{gathered}
$$

where $\beta$ and $k_{0}$ are the lateral wavevector component and the free space wavelength. We see that the optical length of the ppolarized wave can be shorter $\left(\varepsilon_{\|}>\varepsilon_{\perp}\right)$ [longer $\left.\left(\varepsilon_{\|}<\varepsilon_{\perp}\right)\right]$ than that of the s-polarized wave around the wavelength range of phonon polariton excitation, and the optical length difference is enhanced as the pillar height increases.

\subsection{Validity of the ellipsometric values based on effective medium theory by CST microwave studio simulation}

The effective medium theory has been known to work well in the case that the structure is regarded as homogenous medium..$^{49} \mathrm{In}$ this study, the pitch between the pillars is much smaller than the wavelength of interest, while the pillar heights are smaller than the incident wavelength. Thus, we try to validate the calculations utilizing the effective medium theory and CST microwave studio. The results are shown in Fig. 9. In the CST microwave studio simulation, the model is composed of the infinite substrate, pillar array with the hexagonal arrangement, and infinite atmosphere above the structure. The electromagnetically simulated structure is shown in the inset of Fig. 9(d). Note that the mushroom-like head has little influence on the electromagnetic response because the size is small enough as compared to the wavelength of light. There was no significant difference in the calculated results using the effective medium theory (Fresnel equations) and CST microwave studio. Only a slight difference between these two simulations, less than $0.5^{\circ}$, can be found in a magnified image (Fig. 9(c) and (d)). The calculation based on CST Microwave studio reflects Maxwell's equation accurately, without using approximations. ${ }^{50}$ Thus we have concluded that Fresnel calculation based on the effective medium theory well provides sufficient precision compared to full-wave simulation.

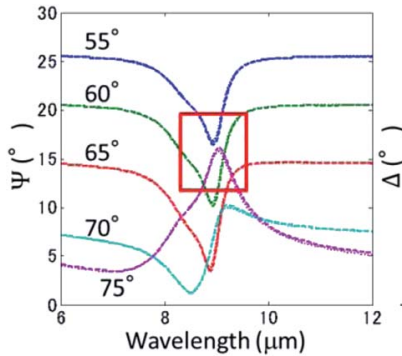

(a)

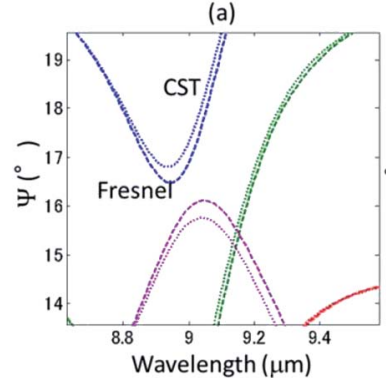

(c)

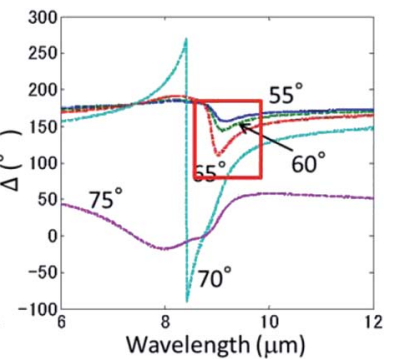

(b)

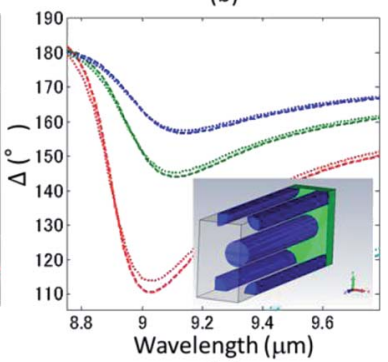

(d)
Fig. 9 Comparison of (a) and (c) ellipsometric spectra $\Psi$ and (b) and (d) $\Delta$ obtained by CST microwave studio (dotted curves) and the effective medium theory (bold dotted curves). The pillar height is set at $200 \mathrm{~nm}$. (c) and (d) are the magnified images of the plots indicated by the red squares in (a) and (b). The inset in (d) depicts the simulation model of $\mathrm{SiO}_{2}$ nanopillars in CST microwave studio.

\section{Conclusions}

To conclude, we have demonstrated a bottom-up approach for the large-scale fabrication of metamaterials consisting of $\mathrm{SiO}_{2}$ nanopillar immersed in air with a cost-effective manner. Selfassembly of PS- $b$-PDMS block copolymers enables to form uniaxial epsilon-near zero metamaterials which are mediated by phonon-polaritons in the mid infrared wavelength region. Unique mushroom-like heads on $\mathrm{SiO}_{x}$ perpendicular cylinders play a key role to realize $\mathrm{SiO}_{2}$ nanopillars having the similar permittivity as that of the fused quartz over a large area. Nanopillars having heights of 80,200 , and $300 \mathrm{~nm}$ are fabricated and the mid-infrared ellipsometry is conducted. The experimental values are well reproduced on the calculated results utilizing the effective medium theory for nanopillars with various heights. The observed agreements indicate an optical anisotropy of the $\mathrm{SiO}_{2}$ nanopillars. Furthermore, the calculation based on the effective medium theory is validated by the CST microwave studio simulation. Due to the intrinsic property of block copolymers, structural parameters, including the size and spacing of $\mathrm{SiO}_{2}$ nanopillars, can be tailored. Such a character may allow us to control the volume fraction of nanopillars and to design metamaterials with hyperbolic permittivity. This study can potentially be used in a variety of optical and thermal applications, with cost-effective manner, and enables to contribute the progress of nanophotonics to benefit our society. 


\section{Conflicts of interest}

There are no conflicts of interest to declare.

\section{Acknowledgements}

The authors are grateful to K. Nakai, K. Kitazumi and Dr H. Yoda for technical supports and fruitful discussions. The authors also acknowledge Dr H. Wakayama and K. Yatsugi for helpful supports. This work was partially supported by the Toyota Technological Institute Nano Technology Hub of the 'Nanotechnology Platform Project' sponsored by the Ministry of Education, Culture, Sports, Science and Technology (MEXT), Japan.

\section{Notes and references}

1 I. Liberal and N. Engheta, Nat. Photonics, 2017, 11, 149.

2 G. Castaldi, S. Savoia, V. Galdi, A. Alù and N. Engheta, Phys. Rev. B: Condens. Matter Mater. Phys., 2012, 86, 115123.

3 A. Alù, M. G. Silveirinha, A. Salandrino and N. Engheta, Phys. Rev. B: Condens. Matter Mater. Phys., 2007, 75, 155410.

4 B. Edwards, A. Alù, M. E. Young, M. Silveirinha and N. Engheta, Phys. Rev. Lett., 2008, 100, 033903.

5 R. Liu, Q. Cheng, T. Hand, J. J. Mock, T. J. Cui, S. A. Cummer and D. R. Smith, Phys. Rev. Lett., 2008, 100, 023903.

6 S. Feng and K. Halterman, Phys. Rev. B: Condens. Matter Mater. Phys., 2012, 86, 165103.

7 S. Vassant, A. Archambault, F. Marquier, F. Pardo, U. Gennser, A. Cavanna, J. L. Pelouard and J. J. Greffet, Phys. Rev. Lett., 2012, 109, 237401.

8 L. Sun, S. Feng and X. Yang, Appl. Phys. Lett., 2012, 101, 241101.

9 J. Luo, P. Xu, H. Chen, B. Hou, L. Gao and Y. Lai, Appl. Phys. Lett., 2012, 100, 221903.

10 L. V. Alekseyev, E. E. Narimanov, T. Tumkur, H. Li, Y. A. Barnakov and M. A. Noginov, Appl. Phys. Lett., 2010, 97, 131107.

11 P. N. Dyachenko, S. Molesky, A. Y. Petrov, M. Störmer, T. Krekeler, S. Lang, M. Ritter, Z. Jacob and M. Eich, Nat. Commun., 2016, 7, 11809.

12 J. Gao, L. Sun, H. Deng, C. J. Mathai, S. Gangopadhyay and X. Yang, Appl. Phys. Lett., 2013, 103, 051111.

13 D. P. Lyvers, J. M. Moon, A. V. Kildishev, V. M. Shalaev and A. Wei, ACS Nano, 2008, 2, 2569-2576.

14 L. Dusan, J. G. Shapter, J. G. Mitchell and N. H. Voelcker, Nanotechnology, 2005, 16, 2275.

15 E. Paul, W. R. Hendren, R. Atkinson, G. A. Wurtz, W. Dickson, A. V. Zayats and R. J. Pollard, Nanotechnology, 2006, 17, 5746.

16 A. V. Kabashin, P. Evans, S. Pastkovsky, W. Hendren, G. A. Wurtz, R. Atkinson, R. Pollard, V. A. Podolskiy and A. V. Zayats, Nat. Mater., 2009, 8, 867-871.

17 P. Ginzburg, F. J. Rodríguez Fortuño, G. A. Wurtz, W. Dickson, A. Murphy, F. Morgan, R. J. Pollard, et al., Opt. Express, 2013, 21, 14907-14917.
18 P. Li, X. Yang, T. W. W. Maß, J. Hanss, M. Lewin, A.-K. U. Michel, M. Wuttig and T. Taubner, Nat. Mater., 2016, 15, 870-875.

19 R. Hillenbrand, T. Taubner and F. Keilmann, Nature, 2002, 418, 159-162.

20 J. D. Caldwell, L. Lindsay, V. Giannini, I. Vurgaftman, T. L. Reinecke, S. A. Maier and O. J. Glembocki, Nanophotonics, 2015, 4, 44-68.

21 F. Neubrech, D. Weber, D. Enders, T. Nagao and A. Pucci, J. Phys. Chem. C, 2010, 114, 7299-7301.

22 J.-J. Greffet, R. Carminati, K. Joulain, J.-P. Mulet, S. Mainguy and Y. Chen, Nature, 2002, 416, 61-64.

23 K. Joulain, J.-P. Mulet, F. Marquier, R. Carminati and J.-J. Greffet, Surf. Sci. Rep., 2005, 57, 59-112.

24 B. J. Lee and Z. M. Zhang, J. Appl. Phys., 2006, 100, 063529.

25 Y. Zhai, Y. Ma, S. N. David, D. Zhao, R. Lou, G. Tan, R. Yang and X. Yin, Science, 2017, 355, 1062-1066.

26 K. Ito, K. Nishikawa, A. Miura, H. Toshiyoshi and H. Iizuka, Nano Lett., 2017, 17, 4347-4353.

27 J. Kyoung, D. J. Park, S. J. Byun, J. Lee, S. B. Choi, S. Park and S. W. Hwang, Opt. Express, 2014, 22, 31875-31883.

28 W. Cai, D. A. Genov and V. M. Shalaev, Phys. Rev. B: Condens. Matter Mater. Phys., 2005, 72, 193101.

29 M. P. Schwartz, S. D. Alvarez and M. J. Sailor, Anal. Chem., 2007, 79, 327-334.

30 X. H. Zhang, J. F. Douglas and R. L. Jones, Soft Matter, 2012, 8, 4980-4987.

31 A. H. Gröschel and A. H. E. Müller, Nanoscale, 2015, 7, 11841-11876.

32 Y. Choo, P. W. Majewski, M. Fukuto, C. O. Osuji and K. G. Yager, Nanoscale, 2018, 1, 416-427.

33 X. D. Gu, I. Gunkel, A. Hexemer, W. Y. Gu and T. P. Russell, Adv. Mater., 2014, 26, 273-281.

34 H. K. Choi, J. Gwyther, I. Manners and C. A. Ross, ACS Nano, 2012, 6, 8342-8348.

35 S. Samant, J. Strzalka, K. G. Yager, K. Kisslinger, D. Grolman, M. Basutkar, N. Salunke, G. Singh, B. Berry and A. Karim, Macromolecules, 2016, 49, 8633-8642.

36 C. M. Bates, M. J. Maher, D. W. Janes, C. J. Ellison and C. G. Willson, Macromolecules, 2013, 47, 2-12.

37 S. O. Kim, H. H. Solak, M. P. Stoykovich, N. J. Ferrier, J. J. de Pablo and P. F. Nealey, Nature, 2003, 424, 411-414.

38 J. Y. Sik, J. H. Lee, J. Y. Lee and C. A. Ross, Nano Lett., 2010, 10, 3722-3726.

39 J. G. Son, K. W. Gotrik and C. A. Ross, ACS Macro Lett., 2012, 1, 1279-1284.

40 G. E. Bhoje, T. Koga, M. K. Endoh, K. Kumar and M. Stamm, Soft Matter, 2014, 10, 7753-7761.

41 Y. Yamada, K. Ito, A. Miura, M. Harada, T. Matsunaga, Y. Kato, T. Matsui, H. Iizuka and H. Wakayama, Eur. Polym. J., 2018, 107, 96-104.

42 Y. Li, X. Bai, T. Yang, H. Luo and C.-W. Qiu, Nat. Commun., 2018, 9, 273.

43 Y. Li, K.-J. Zhu, Y.-G. Peng, W. Li, T. Yang, H.-X. Xu, H. Chen, X.-F. Zhu, S. Fan and C.-W. Qiu, Nat. Mater., 2019, 18, 48-54.

$44 \mathrm{H}$. Fujiwara, in Spectroscopic Ellipsometry: Principles and Applications, Wiley, UK, 2007. 
45 Q. Xia, K. J. Morton, R. H. Austin and S. Y. Chou, Nano Lett., 2008, 8, 3830-3833.

46 J. Hopkins and J. P. S. Badyal, Macromolecules, 1994, 27, 5498-5503.

47 J. J. Saarinen, S. M. Weiss, P. M. Fauchet and J. E. Sipe, J. Appl. Phys., 2008, 104, 013103.
48 R. Kitamura, L. Pilon and M. Jonas, Appl. Opt., 2007, 46, 8118-8133.

49 T. C. Choy, in Effective Medium Theory, ed. J. Birman, S. F. Edwards, R. Friend, M. Rees, D. Sherrington and G. Veneziano, Oxford Univ. Press, UK, 2nd edn, 2015.

50 N. C. Dyck, R. C. Denomme and P. M. Nieva, J. Phys. Chem. C, 2011, 115, 15225-15233. 